BMJ Open

Diabetes

Research

\& Care

\title{
Association of prestroke glycemic status with stroke mortality
}

\author{
Paola Forti (D) , ${ }^{1}$ Fabiola Maioli, ${ }^{2}$ Valeria Nativio, ${ }^{2}$ Lorenzo Maestri, ${ }^{1}$ Maura Coveri, ${ }^{2}$ \\ Marco Zoli ${ }^{1}$
}

To cite: Forti P, Maioli F, Nativio $\mathrm{V}$, et al. Association of prestroke glycemic status with stroke mortality. BMJ Open Diab Res Care 2020;8:e000957. doi:10.1136/ bmjdrc-2019-000957

Received 5 0ctober 2019 Revised 23 December 2019 Accepted 4 January 2020

\section{Check for updates}

C) Author(s) (or their employer(s)) 2020. Re-use permitted under CC BY-NC. No commercial re-use. See rights and permissions. Published by BMJ.

${ }^{1}$ Department of Medical and Surgical Sciences, University of Bologna, Bologna, EmiliaRomagna, Italy

${ }^{2}$ Medical Department of Integrated Care Models, Maggiore Hospital Carlo Alberto Pizzardi, Bologna, EmiliaRomagna, Italy

Correspondence to Dr Paola Forti; paola.forti@unibo.it

\section{ABSTRACT}

Objective The role of diabetes as a predictor of mortality after stroke remains uncertain, and there are very few data for pre-diabetes. This study investigated the association of pre-diabetes and diabetes with 30-day and 1-year mortality after ischemic stroke (IS) and primary intracerebral hemorrhage (ICH).

Research design and methods Between 2006 and 2013, 2076 patients with IS and 586 patients with ICH (median age 79 ) were admitted to hospital within 24 hours after stroke onset and were treated in a stroke unit, where they underwent measurement of glycated hemoglobin (HbA1c). Diabetes was retrospectively defined based on medical history, diagnosis during hospital stay or $\mathrm{HbA1C}$ $\geq 6.5 \%$ (48 mmol $/ \mathrm{mol})$. Pre-diabetes was defined as $\mathrm{HbA1c}$ of $5.7 \%-6.4 \%$ (39-47 mmol/mol). Stroke severity was measured using the National Institutes of Health Stroke Scale (NIHSS). HRs were used to test the association of pre-diabetes and diabetes with 30-day and 1-year mortality after stroke onset.

Results Among patients with IS, 830 had pre-diabetes and 632 had diabetes; 280 died within 30 days and the other 77 within 1 year. Among patients with $\mathrm{ICH}, 106$ had pre-diabetes and 56 had diabetes; 150 died within 30 days and the other 92 within 1 year. In both stroke subtypes, pre-diabetes and diabetes were associated with higher 30-day mortality. In IS, however, the association was limited to patients with prestroke disability and very severe stroke. At NIHSS 25, HR was 1.58 (95\% Cl 1.07 to 2.35) for pre-diabetes and 1.67 (95\% $\mathrm{Cl} 1.14$ to 2.46$)$ for diabetes compared with normoglycemia. In ICH, the association was limited to women for pre-diabetes ( $\mathrm{HR} 1.93,95 \% \mathrm{Cl} 1.15$ to 3.24 ) and to men for diabetes (HR 1.78, 95\% $\mathrm{Cl} 1.02$ to 3.12). Prestroke glycemic status was unrelated to 1 -year mortality.

Conclusions Both pre-diabetes and diabetes predict short-term mortality after acute stroke, but the association varies depending on both prestroke and strokerelated characteristics. These findings may explain the heterogeneous results obtained by previous studies.

\section{INTRODUCTION}

Diabetes is an acknowledged risk factor for mortality in the general population. ${ }^{1}$ Similar evidence is growing for pre-diabetes, ${ }^{2}$ the intermediate metabolic state between normal glucose metabolism and diabetes. ${ }^{3}$ Both conditions are highly prevalent in patients with acute stroke. ${ }^{4}$ However, the association of diabetes with mortality after stroke remains

\section{Significance of this study}

What is already known about this subject?

- Both pre-diabetes and diabetes are highly prevalent in patients with acute stroke, but their predictive role in mortality after stroke is not yet clearly defined.

What are the new findings?

- Both pre-diabetes and diabetes predict 30-day mortality after acute stroke, but the association noticeably varies depending on both prestroke and stroke-related characteristics of patients.

How might these results change the focus of research or clinical practice?

- These findings contribute to explaining the heterogeneity of results obtained by previous studies of this topic.

- The findings also prompt further investigations of the mechanisms by which prestroke and stroke-related characteristics of the individual patient can modify the prognostic value of prestroke glycemic status for stroke mortality.

uncertain, ${ }^{5}$ and data for pre-diabetes are too scant to draw a conclusion. ${ }^{6-9}$ Clarifying this issue is important because predictors of stroke mortality are useful to inform clinical decision-making or to project the need for healthcare services. Moreover, pre-diabetes and diabetes are both potentially modifiable risk factors.

This study investigated the association of pre-diabetes and diabetes with 30-day and 1-year mortality after acute stroke.

\section{RESEARCH DESIGN AND METHODS \\ Subjects}

The study sample was retrospectively drawn from a cohort of 2731 patients aged $\geq 18$ year who, between January 2006 and December 2013, were consecutively admitted to the Emergency Room of the Maggiore Hospital (Bologna, Italy) within 24 hours after onset of ischemic stroke (IS) or primary intracerebral hemorrhage (ICH). Diagnosis was based on clinical and neuroradiological data. All 
patients were transferred to the local stroke unit (SU) and treated according to institutional guidelines for stroke care. The cohort did not include (1) patients with ICH associated with aneurysm, arteriovenous malformation or other structural lesions; and (2) any patient who, before SU admission, needed treatment in intensive care units or spent more than 48 hours in the emergency department. At SU admission, the patient or their legally authorized representatives were requested to provide written informed consent for the data contained in their medical chart and information about long-term vital status as obtained from public sources to be used in future research studies. For this study we excluded 21 patients due to refusal/inability to provide informed consent and 40 patients with missing laboratory data. Excluded patients did not differ by age and sex from included patients.

All information used in this report was abstracted from patients' medical charts.

\section{Prestroke glycemic status}

Glycated hemoglobin (HbAlc) was determined the morning after SU admission as part of routine biochemistry tests performed on a venous blood sample drawn after an overnight fast. All measurements were performed using automated methods at the same central laboratory (Diabetes Control and Complications Trial aligned results). Diabetes was defined as known preadmission diagnosis (self-report, evidence from available medical records of previous diagnosis of diabetes, use of antidiabetic agents), clinical diagnosis made during $\mathrm{SU}$ stay or admission $\mathrm{HbAlc} \geq 6.5 \%$ (48 $\mathrm{mmol} / \mathrm{mol})$; prediabetes was defined as admission HbAlc of $5.7 \%-6.4 \%$ $(39-47 \mathrm{mmol} / \mathrm{mol}){ }^{3}$

\section{Covariates}

Choice of covariates was based on literature about stroke mortality predictors. ${ }^{10}{ }^{11}$ Prestroke characteristics included age, sex, heart disease (any history of coronary heart disease or congestive heart failure), history of cancer or hematologic malignancy in the previous 5 years, and pre-existing functional impairment (preadmission score $\geq 1$ on the modified Rankin Scale ${ }^{12}$ ). Stroke characteristics included admission clinical severity measured with the National Institutes of Health Stroke Scale (NIHSS), ${ }^{13}$ thrombolytic treatment, and lacunar etiology ${ }^{14}$ for IS; and admission clinical severity measured with the Glasgow Coma Scale, ${ }^{16}$ non-lobar versus lobar location of hematoma, ${ }^{17}$ and intraventricular extension on admission CT head for ICH.

\section{Outcome}

The outcome was all-cause mortality at 30 days and 1 year after stroke onset. Data were obtained from the Regional Mortality Registry.

\section{Statistics}

Data are reported as median (IQR) or n (\%). Differences in baseline characteristics by prestroke glycemic status were tested using $\chi^{2}$ test for categorical variables and Kruskal-Wallis test for continuous variables.

The association of pre-diabetes and diabetes with mortality was studied using Cox proportional hazard regression. Analyses for 1-year mortality included only patients still alive at day 30. Results were reported as HR with 95\% CI. Cox models were adjusted for both prestroke and stroke-related characteristics. Regardless of stroke subtype, prestroke characteristics included age, sex, functional impairment, and history of heart disease; models for 1-year mortality additionally included history of cancer or hematologic malignancy. Stroke-related characteristics depended on stroke subtype. Analyses were performed with R V.3.5.3 software. Significance was set at a $p$ value of 0.050 (two-tailed).

\section{RESULTS}

\section{Baseline characteristics}

The final cohort included 2076 patients with IS (median age 79 , range $19-100$ years) and 586 with ICH (median age 79, range 34-104years). Among patients with IS, $40.0 \%$ had pre-diabetes and $30.4 \%$ had diabetes. Among patients with ICH, 35.3\% had pre-diabetes and 23.5\% had diabetes. The number of diabetes cases retrospectively identified by HbAlc was 136 in IS and 30 in ICH. Baseline characteristics by stroke type and prestroke glycemic status are summarized in table 1 .

\section{IS mortality}

Among patients with IS, 280 died within 30 days. Unadjusted mortality rate was unrelated to prestroke glycemic status $(11.6 \%$ for normoglycemic, $13.4 \%$ for pre-diabetes, and $15.5 \%$ for diabetes; $\mathrm{p}=0.125)$. In multivariableadjusted Cox models, however, there was a significant three-way interaction involving prestroke glycemic status, admission NIHSS score, and disability $(\mathrm{p}<0.001)$. Among patients without prestroke disability, the risk of 30-day mortality did not change in pre-diabetes (42 deaths out of 545 patients, $7.7 \%$; HR, 1.05 ; $95 \%$ CI 0.55 to 1.65 ) and diabetes (30 deaths out of 376 patients, $8.0 \%$; HR, 1.33; $95 \%$ CI 0.40 to 1.39 ) compared with normoglycemia (21 deaths out of 373 patients, $5.6 \%$ ); estimates were independent of NIHSS score. Conversely, among patients with prestroke disability, the risk of 30-day mortality was associated with both pre-diabetes and diabetes, but the strength of the associations depended on stroke severity: estimates for HRs increased with increasing NIHSS score and became significant only for NIHSS $\geq 25$ (figure 1 ). For pre-diabetes, HR went from 1.58 (95\% CI 1.07 to 2.35 ) at NIHSS 25 to 2.12 (95\% CI 1.18 to 3.82 ) at NIHSS 35. The corresponding HRs for diabetes went from 1.67 (95\% CI 1.14 to 2.46 ) to 2.67 (95\% CI 1.53 to 4.68 ).

Information about 1-year mortality was unavailable for 13 of the 1796 IS survivors. Unadjusted 1-year mortality rates varied with prestroke glycemic status $(\mathrm{p}=0.005)$ : $18.0 \%$ for normoglycemia (102 deaths out of 538 patients with normoglycemia), $15.7 \%$ for pre-diabetes (112 deaths 
Table 1 Baseline characteristics of patients with acute stroke by prestroke glycemic status

\begin{tabular}{|c|c|c|c|c|}
\hline & Normoglycemia & Pre-diabetes & Diabetes & $P$ value \\
\hline \multicolumn{5}{|l|}{ Ischemic stroke } \\
\hline $\mathrm{n}$ & 614 & 830 & 632 & \\
\hline Female & $315(51.3)$ & $470(56.6)$ & $317(50.2)$ & 0.028 \\
\hline Age, years & $79(68-86)$ & $79(71-85)$ & $79(71-85)$ & 0.466 \\
\hline Disability & $241(39.3)$ & $285(34.3)$ & $256(40.5)$ & 0.034 \\
\hline Heart disease & $115(18.7)$ & $175(21.1)$ & $173(27.4)$ & 0.001 \\
\hline Cancer or hematologic malignancy & $63(10.3)$ & $110(13.3)$ & $85(13.4)$ & 0.151 \\
\hline NIHSS score & $6(3-14)$ & $6(3-15)$ & $7(3-16)$ & 0.376 \\
\hline Fibrinolysis & $58(9.4)$ & $76(9.2)$ & $35(5.5)$ & 0.016 \\
\hline Non-lacunar subtype & $485(79.0)$ & $682(82.2)$ & $482(76.3)$ & 0.021 \\
\hline \multicolumn{5}{|l|}{ Intracerebral hemorrhage } \\
\hline $\mathrm{n}$ & 136 & 106 & 56 & \\
\hline Female & $136(56.4)$ & $106(51.2)$ & $56(40.6)$ & 0.012 \\
\hline Age, years & $78(69-84)$ & 79 (72-84) & $78(72-84)$ & 0.419 \\
\hline Disability & $97(40.2)$ & $73(35.3)$ & $56(40.6)$ & 0.478 \\
\hline Heart disease & $20(8.3)$ & $24(11.6)$ & 27 (19.6) & 0.005 \\
\hline Cancer or hematologic malignancy & $25(10.4)$ & $25(12.1)$ & $20(14.5)$ & 0.491 \\
\hline GCS score & $14(10-15)$ & $14(11-15)$ & $14(10-15)$ & 0.737 \\
\hline Non-lobar location & $141(58.5)$ & $130(63.1)$ & $92(66.7)$ & 0.268 \\
\hline Intraventricular extension & $79(32.8)$ & $85(41.3)$ & $45(32.6)$ & 0.737 \\
\hline
\end{tabular}

Data are median (IQR) or $\mathrm{n}(\%)$.

GCS, Glasgow Coma Scale; NIHSS, National Institutes of Health Stroke Scale.

out of 714 with pre-diabetes), and $23.0 \%$ for diabetes (122 deaths out of 531 patients). In multivariable-adjusted Cox models, however, the risk of 1-year mortality was unrelated to pre-diabetes (HR, 0.90; $95 \%$ CI 0.69 to 1.18 ) and diabetes (HR, $1.30 ; 95 \%$ CI 0.90 to 1.70$)$.

\section{ICH mortality}

Among patients with ICH, 150 died within 30 days. Unadjusted mortality rate was unrelated to prestroke glycemic status $(24.1 \%$ for normoglycemia, $25.1 \%$ for pre-diabetes, and $29.0 \%$ for diabetes; $\mathrm{p}=0.562$ ). In multivariableadjusted Cox models, however, there was a significant interaction between prestroke glycemic status and sex $(\mathrm{p}<0.001)$. As shown in figure 2,30 -day mortality was associated with pre-diabetes but not diabetes in women, and with diabetes but not pre-diabetes in men.

Information about 1-year mortality was unavailable for 4 of the 436 ICH survivors. Unadjusted 1-year mortality rate was unrelated to glycemic status $(\mathrm{p}=0.338)$ : $23.9 \%$ for normoglycemia (43 deaths out of 180 patients), $21.4 \%$ for pre-diabetes (33 deaths out of 154 patients), and $16.3 \%$ for diabetes (16 deaths out of 98 patients). In multivariable-adjusted Cox models, the results did not change for either pre-diabetes (HR, 0.92; 95\% CI 0.58 to 1.48 ) and diabetes (HR, 0.69; 95\% CI 0.38 to 1.25 ) compared with normoglycemia.

\section{DISCUSSION}

The main finding of this study is that both pre-diabetes and diabetes predict 30-day mortality after acute IS, but only in patients with prestroke disability and greater neurologic severity on admission. The study also found an association of 30-day mortality after ICH with prediabetes in women and diabetes in men. No association was found between prestroke glycemic status and 1-year mortality in either stroke type.

Previous literature on diabetes as a mortality predictor in acute stroke is large but inconsistent. ${ }^{5}$ For IS, some studies reported an association of diabetes with both short-term (up to 30 days) and long-term mortality, ${ }^{18}$ but others did not. ${ }^{11} 1920$ For ICH, a meta-analysis of 18 cohort studies found a weak association of diabetes with short-term mortality, but analyses were not adjusted for possible confounders. ${ }^{21}$ Individual cohort studies controlling for ICH severity did not find any association between short-term mortality and diabetes. ${ }^{22}{ }^{23}$ It is also unclear whether diabetes is a predictor of long-term mortality after ICH. ${ }^{22}$ 24-27

Literature about pre-diabetes and stroke mortality is scant and based on small cohorts. ${ }^{6-9}$ No association was reported with short-term mortality after IS. ${ }^{8}$ In studies of long-term mortality, a positive association was reported in IS ${ }^{7}$ but not in stroke from any cause ${ }^{6}$ and ICH. ${ }^{9}$ As all these studies used blood glucose criteria for pre-diabetes 


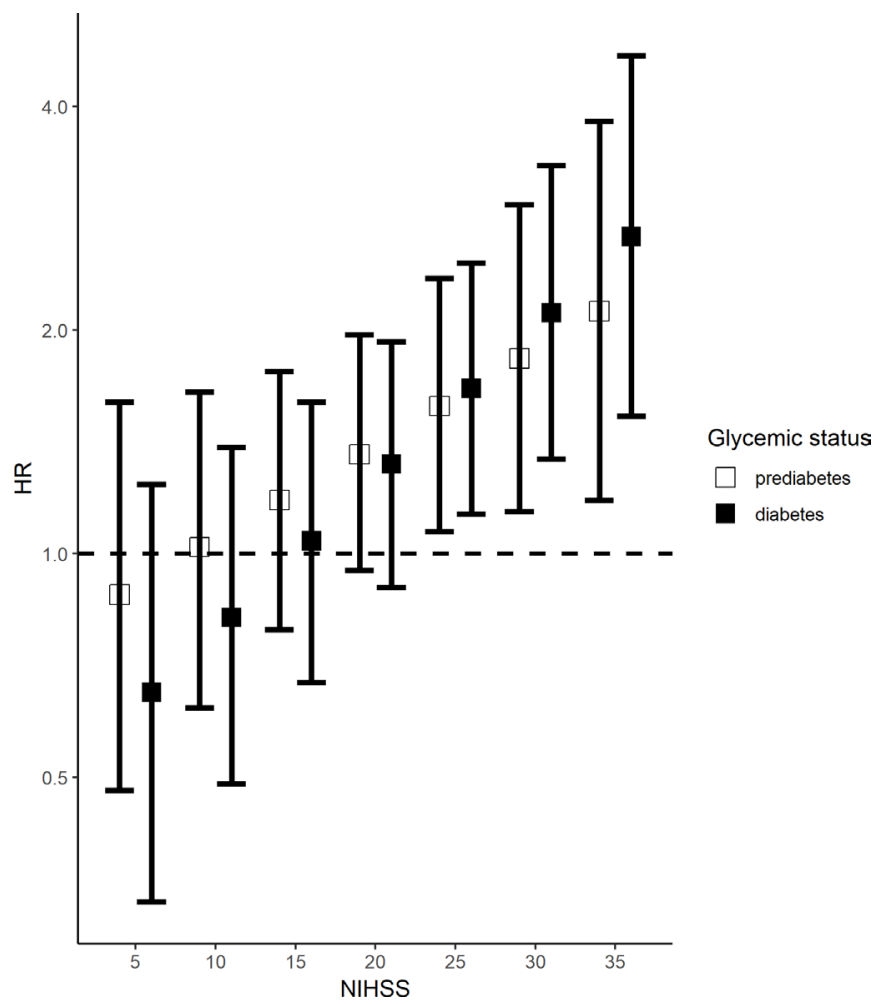

Figure 1 Association of prestroke glycemic status and 30-day mortality after ischemic stroke across values of admission NIHSS score in patients with prestroke disability. $\mathrm{HR}$ and $95 \% \mathrm{Cl}$ were estimated from Cox models adjusted for age, sex, history of heart disease, thrombolytic treatment and lacunar etiology. The number of deaths/number of patients was 50/241 for normoglycemia (reference group), $69 / 288$ for pre-diabetes and 68/256 for diabetes. NIHSS, National Institutes of Health Stroke Scale.

diagnosis, stress hyperglycemia may have biased the estimates.

Only one study reported an interaction between diabetes and stroke severity in mortality prediction models: diabetes predicted both short-term and longterm mortality after ICH, but only in non-comatose patients, possibly because coma obscured the prognostic role of weaker predictors. ${ }^{28}$

In this study, conversely, pre-diabetes and diabetes acted as predictors of short-term mortality only in patients with IS with prestroke disability and very severe stroke. Taking prestroke disability as an indicator of poor prestroke health status, it can be hypothesized that chronic dysglycemia is a significant mortality predictor only in the most compromised and vulnerable patients. An alternative explanation relies on the 'score what you see' rule, which informs use of the NIHSS in order to gain reproducibility. ${ }^{13}$ As no distinction is made between new and pre-existent deficits, the predictive ability of NIHSS for mortality may be lower in patients with pre-existent motor or cognitive impairments, allowing weaker predictors, such as chronic hyperglycemia, to emerge.

In some cohorts with acute stroke from any cause, diabetes was associated with mortality only in men. ${ }^{29-31}$ In
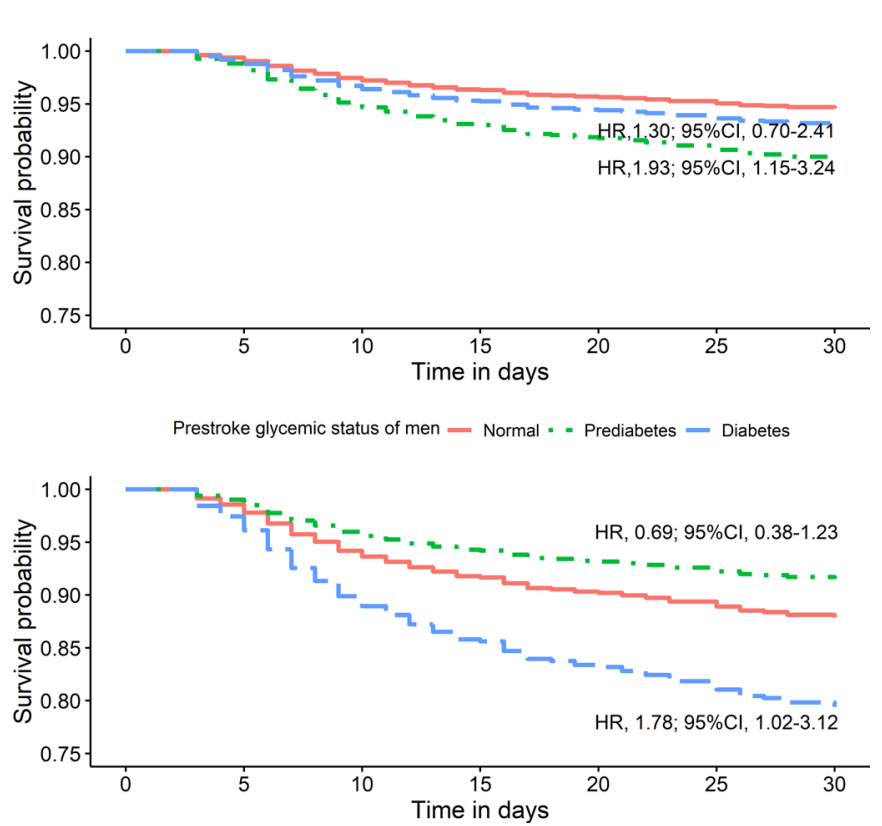

Figure 2 Association of prestroke glycemic status with 30-day mortality after intracerebral hemorrhage in women (top) and men (bottom). For women, the number of deaths/ number of patients was 30/136 for normoglycemia (reference group), 31/106 for pre-diabetes, and 16/56 for diabetes. For men, the number of deaths/number of patients was 28/105 for normoglycemia (reference group), 21/101 for pre-diabetes, and 24/82 for diabetes. HR and $95 \% \mathrm{Cl}$ were estimated from Cox models adjusted for age, history of heart disease, Glasgow Coma Scale score, hematoma location, and intraventricular extension of hemorrhage.

our study, however, a modifying effect of sex was observed only for prediction of short-term mortality after ICH: prediabetes was prognostic in women only and diabetes in men only. This pattern might result from underdiagnosis of pre-diabetes in men, which is a known pitfall of the HbA1c criterion, ${ }^{32}$ and of diabetes in women, among whom impaired glucose tolerance (requiring a glucose challenge for diagnosis) is more frequent than persistent elevation of blood glucose (detectable by a simple fasting measurement) ${ }^{33}$ Moreover, risk factors and their mechanisms of brain injury in ICH are different from those in IS (hypertension, in particular, plays a much relevant causal role), and hematoma characteristics are a more important predictor of short-term mortality than severity measured by NIHSS. ${ }^{10}$ All these considerations suggest caution in the interpretation of our findings in ICH and their possible clinical usefulness.

The major strengths of this study are the paucity of similar data for pre-diabetes, provision of data for different stroke subtypes, and use of HbAlc for a systematic screening of pre-diabetes and unknown diabetes cases. Diabetes goes often undiagnosed in patients with stroke, and awareness of pre-diabetes remains very low in all clinical settings. ${ }^{34}{ }^{35}$ Since 2010 , HbA1c has been considered a valid alternative to the traditional blood glucose criteria. ${ }^{3}$ Although HbAlc and blood glucose 
criteria are not fully concordant, ${ }^{32} \mathrm{HbAlc}$ is a reasonable choice in acute stroke because it is easy to perform and, differently from blood glucose criteria, is unaffected by acute-phase reaction. ${ }^{36}$

This study also has several limitations. First is its retrospective design which does not allow for identification of causal relationships. Literature, however, suggests that prestroke glycemic status may affect prognosis of acute stroke by several mechanisms. Diabetes can increase the risk of poststroke infections and interfere with neuronal repair. ${ }^{28}{ }^{37}$ Hyperglycemia can worsen brain ischemic damage (local toxicity from increased anaerobic metabolism, interference with fibrinolytic treatment) and increase the risk of hematoma expansion and perihematomal edema (stimulation of proinflammatory response, disruption of the blood-brain barrier). ${ }^{515}$ These adverse effects, however, are greater for acute than for chronic hyperglycemia. ${ }^{5}$ This might explain the relatively weak associations found in this study.

Second, the study sample comes from a single hospital and may not be representative of other institutions. Moreover, our findings may not be applicable to patients with very severe stroke because we excluded patients requiring intensive care treatment immediately on hospital admission and we lack data for the patients who died before reaching the hospital. On the basis of our findings, however, inclusion of these patients should have strengthened the predictive ability of diabetes for short-term mortality in patients with prestroke disability. Third, data about prestroke characteristics were based on information as recorded in patients' medical charts. Fourth, the retrospective identification of pre-diabetes and unknown diabetes relied on a single $\mathrm{HbAlc}$ measurement. Finally, we lack information about diabetes type, cause of death, infarct size and variations in glycemic status after discharge.

In conclusion, this study shows that pre-diabetes and diabetes are both associated with short-term mortality after acute stroke, but only in subsets of patients with peculiar characteristics. Our findings contribute to explaining the heterogeneous results obtained by previous studies on this issue, and underscore the need for further investigations of the mechanisms by which prestroke and stroke-related conditions can modify the prognostic value of glycemic status for stroke mortality.

Contributors PF conceived and designed the study, proposed and performed the statistical analyses, contributed to literature search, drafted the report, and reviewed/edited the manuscript for important intellectual content. FM conceived and designed the study, obtained the data, provided administrative, technical, and material support, contributed to the literature search, and reviewed/edited the manuscript for important intellectual content. VN and LM obtained the data, contributed to literature search, and reviewed/edited the manuscript for important intellectual content. MC and MZ provided administrative and material support, contributed to the literature search, and reviewed/edited the manuscript for important intellectual content. PF is the guarantor of the work, and as such had full access to all the data in the study and takes responsibility for the integrity of the data and the accuracy of the data analysis. The principal author certifies that all the persons listed as authors meet the authorship criteria and participated sufficiently in the work to take public responsibility for the content, including participation in the concept, design, analysis, writing, or revision of the manuscript.
Funding This work was supported by the Basic Oriented Research grant from the University of Bologna.

Competing interests None declared.

Patient consent for publication Not required.

Ethics approval The Maggiore Hospital Ethics Committee approved the study.

Provenance and peer review Not commissioned; externally peer reviewed.

Data availability statement All data relevant to the study are included in the article.

Open access This is an open access article distributed in accordance with the Creative Commons Attribution Non Commercial (CC BY-NC 4.0) license, which permits others to distribute, remix, adapt, build upon this work non-commercially, and license their derivative works on different terms, provided the original work is properly cited, appropriate credit is given, any changes made indicated, and the use is non-commercial. See: http://creativecommons.org/licenses/by-nc/4.0/.

\section{ORCID iD}

Paola Forti http://orcid.org/0000-0001-6452-8697

\section{REFERENCES}

1 Röckl S, Brinks R, Baumert J, et al. All-Cause mortality in adults with and without type 2 diabetes: findings from the National health monitoring in Germany. BMJ Open Diabetes Res Care 2017;5:e000451.

2 Huang Y, Cai X, Mai W, et al. Association between prediabetes and risk of cardiovascular disease and all cause mortality: systematic review and meta-analysis. BMJ 2016;355:i5953.

3 American Diabetes Association. 2. Classification and Diagnosis of Diabetes: Standards of Medical Care in Diabetes-2018. Diabetes Care 2018;41:S13-27.

4 Fonville S, Zandbergen AAM, Koudstaal PJ, et al. Prediabetes in patients with stroke or transient ischemic attack: prevalence, risk and clinical management. Cerebrovasc Dis 2014;37:393-400.

5 Lau L-H, Lew J, Borschmann K, et al. Prevalence of diabetes and its effects on stroke outcomes: A meta-analysis and literature review. $J$ Diabetes Investig 2019;10:780-92.

6 Hyvärinen M, Qiao Q, Tuomilehto J, et al. Hyperglycemia and stroke mortality: comparison between fasting and 2-h glucose criteria. Diabetes Care 2009;32:348-54.

7 Jia Q, Liu G, Zheng H, et al. Impaired glucose regulation predicted 1 -year mortality of Chinese patients with ischemic stroke: data from abnormal glucose regulation in patients with acute stroke across China. Stroke 2014;45:1498-500.

8 Roquer J, Giralt-Steinhauer E, Cerdà G, et al. Glycated hemoglobin value combined with initial glucose levels for evaluating mortality risk in patients with ischemic stroke. Cerebrovasc Dis 2015;40:244-50.

9 Sun S, Pan Y, Zhao X, et al. The association between impaired glucose regulation and prognosis of Chinese patients with intracerebral hemorrhage. Sci Rep 2016;6:36220.

10 Hemphill JC, Greenberg SM, Anderson CS, et al. Guidelines for the management of spontaneous intracerebral hemorrhage. Stroke 2015;46:2032-60.

11 Saposnik G, Kapral MK, Liu Y, et al. IScore. Circulation 2011;123:739-49.

12 van Swieten JC, Koudstaal PJ, Visser MC, et al. Interobserver agreement for the assessment of handicap in stroke patients. Stroke 1988;19:604-7.

13 Lyden P. Using the National Institutes of health stroke scale: a cautionary tale. Stroke 2017;48:513-9.

14 Adams HP, Bendixen BH, Kappelle LJ, et al. Classification of subtype of acute ischemic stroke. definitions for use in a multicenter clinical trial. TOAST. trial of ORG 10172 in acute stroke treatment. Stroke 1993;24:35-41.

15 Robbins NM, Swanson RA. Opposing effects of glucose on stroke and reperfusion injury: acidosis, oxidative stress, and energy metabolism. Stroke 2014;45:1881-6.

16 Teasdale G, Jennett B. Assessment of coma and impaired consciousness. A practical scale. Lancet 1974;2:81-4.

17 Samarasekera N, Fonville A, Lerpiniere C, et al. Influence of intracerebral hemorrhage location on incidence, characteristics, and outcome: population-based study. Stroke 2015;46:361-8.

18 Luitse MJA, Biessels GJ, Rutten GEHM, et al. Diabetes, hyperglycaemia, and acute ischaemic stroke. Lancet Neurol 2012:11:261-71.

19 Kamalesh M, Shen J, Eckert GJ. Long term postischemic stroke mortality in diabetes: a veteran cohort analysis. Stroke 2008;39:2727-31. 
20 O'Donnell MJ, Fang J, D'Uva C, et al. The plan score: a bedside prediction rule for death and severe disability following acute ischemic stroke. Arch Intern Med 2012;172:1548-56.

21 Boulanger M, Poon MTC, Wild SH, et al. Association between diabetes mellitus and the occurrence and outcome of intracerebra hemorrhage. Neurology 2016;87:870-8.

22 Liebkind R, Gordin D, Strbian D, et al. Diabetes and intracerebra hemorrhage: baseline characteristics and mortality. Eur J Neurol 2018;25:825-32.

23 Saxena A, Anderson CS, Wang X, et al. Prognostic significance of hyperglycemia in acute intracerebral hemorrhage: the INTERACT2 study. Stroke 2016;47:682-8.

24 Hansen BM, Nilsson OG, Anderson H, et al. Long term (13 years) prognosis after primary intracerebral haemorrhage: a prospective population based study of long term mortality, prognostic factors and causes of death. J Neurol Neurosurg Psychiatry 2013;84:1150-5.

25 Muñoz-Rivas N, Méndez-Bailón M, Hernández-Barrera V, et al. Type 2 diabetes and hemorrhagic stroke: a population-based study in Spain from 2003 to 2012. J Stroke Cerebrovasc Dis 2016;25:1431-43

26 Poon MTC, Fonville AF, Al-Shahi Salman R. Long-Term prognosis after intracerebral haemorrhage: systematic review and metaanalysis. J Neurol Neurosurg Psychiatry 2014;85:660-7.

27 Zhang $\mathrm{X}$, Jing J, Zheng $\mathrm{H}$, et al. Prognosis of intracerebral hemorrhage with newly diagnosed diabetes mellitus according to hemoglobin A1c criteria. J Stroke Cerebrovasc Dis 2018;27:1127-33.

28 Passero S, Ciacci G, Ulivelli M. The influence of diabetes and hyperglycemia on clinical course after intracerebral hemorrhage. Neurology 2003;61:1351-6.
29 Eriksson M, Carlberg B, Eliasson M. The disparity in long-term survival after a first stroke in patients with and without diabetes persists: the Northern Sweden MONICA study. Cerebrovasc Dis 2012;34:153-60.

30 Heuschmann PU, Kolominsky-Rabas PL, Misselwitz B, et al. Predictors of in-hospital mortality and attributable risks of death after ischemic stroke: the German stroke registers Study Group. Arch Intern Med 2004;164:1761-8.

31 Soriano-Reixach MM, Vivanco-Hidalgo RM, Ois A, et al. Interaction of sex and diabetes on outcome after ischemic stroke. Front Neurol 2018;9:250

32 Lipska KJ, De Rekeneire N, Van Ness PH, et al. Identifying dysglycemic states in older adults: implications of the emerging use of hemoglobin A1c. J Clin Endocrinol Metab 2010;95:5289-95.

33 Kautzky-Willer A, Harreiter J, Pacini G. Sex and gender differences in risk, pathophysiology and complications of type 2 diabetes mellitus. Endocr Rev 2016;37:278-316.

34 Quinn TJ, Lees KR. Hyperglycaemia in acute stroke--to treat or not to treat. Cerebrovasc Dis 2009;27:148-55.

34 Centers for Disease Control and Prevention (CDC). Awareness of prediabetes--United States, 2005-2010. MMWR Morb Mortal Wkly Rep 2013;62:209-12.

36 Kernan WN. Screening for diabetes after stroke and transient ischemic attack. Cerebrovasc Dis 2013;36:290-1.

37 Arnold SE, Arvanitakis Z, Macauley-Rambach SL, et al. Brain insulin resistance in type 2 diabetes and Alzheimer disease: concepts and conundrums. Nat Rev Neurol 2018;14:168-81. 